\title{
Revised Computational-GOMS Model for Drag Activity
}

\author{
Yongho Lee, Young Joo Jeon, Rohae Myung \\ Department of Industrial Management Engineering, Korea University, Seoul 136-713
}

\begin{abstract}
The existing GOMS model overestimates the performance time of mouse activities because it describes them in a serial sequence. However, parallel movements of eye and hand(eye-hand coordination) have been dominant in mouse activities and this eye-hand coordination is the main factor for the overestimation of performance time. In this study, therefore, the revised CGOMSL model was developed to implement eye-hand coordination to the mouse activity to overcome one of the limitations of GOMS model, the lack of capability for parallel processing. The suggested revised CGOMSL model for drag activity, as an example for one of mouse activities in this study, begins visual search processing before a hand movement but ends the visual search processing with the hand movement in the same time. The results show that the revised CGOMSL model made the prediction of human performance more accurately than the existing GOMS model. In other words, one of the limitations of GOMS model, the incapability of parallel processing, could be overcome with the revised CGOMSL model so that the performance time should be more accurately predicted.
\end{abstract}

Keywords: CGOMSL, Drag activity, Eye-Hand Coordination, Parallel Processing

\section{Introduction}

\subsection{Overview}

시스템이 다양해지고 복잡해짐에 따라 이를 작동하기 위 한 인터페이스는 사용자가 직관적으로 쉽게 조작할 수 있으 며, 사용자의 오류를 최소화 할 수 있는 방향으로 설계되어 야 한다. 이와 같은 사용자 인터페이스(UI; User Interface) 를 개발하기 위해서는 프로토타입을 만들어 평가한 결과를 통해 개선하는 과정이 필수적이다. 이때 인터페이스가 시스 템의 목적에 잘 부합되어 설계되었는지 평가, 분석하는 것 을 사용성 평가(UT; Usability Tests)라고 한다(Bennett et al., 1989). 사용성 평가의 결과는 시스템의 인터페이스 를 개선시키는데 이용되기도 하지만, 사용자에 관한 정보 를 제공하기 때문에 평가된 시스템 외에도 새로운 사용자 인터페이스를 구현하는데 이용된다(Wiecha et al., 1990, Puerta et al., 1997).

전통적으로 사용성 평가는 실제 사용자의 수행 과정의 관
찰을 통해 사용자의 수행도 및 만족도 등을 측정하는 직접 적인 분석 방법이 적용되어 왔다(Galitz, 2002). 실제 사용 자 실험을 통해 얻은 사용성 평가 결과를 바탕으로 기존의 인터페이스를 재설계하고, 이에 대한 사용성 평가 과정을 다 시 거쳐 사용성 문제를 해결하여 시스템의 성능을 향상시키 는 것은 가장 보편적으로 실시되는 사용자 인터페이스 평가 및 설계 방법론이다(Landauer, 1995, Kieras, 1999).

하지만 위와 같은 방법론은 사용자를 훈련시키는 시간과 비용이 많이 소모되고(Card et al., 1983, Bennett et al., 1989), 실험 참가자 집단을 평가할 시스템을 능숙하게 하용 하는 전문가 수준으로 숙련시키기가 어렵다는 단점이 존재 한다(Olson \& Olson, 1990, Kieras., 1996). 따라서 Card et al. (1980, 1983)는 공학적 모델(Engineering Model)의 개념을 사용성 평가에 적용하기 시작하였으며, Gould et al. 1985,1987 )는 공학적 원칙 (Engineering Principal)에 사 용성 원칙을 도입하였다. 그 결과 인터페이스를 디자인하고 평가하는데 강력한 직관을 가지고 분석적인 접근이 가능한 모델 기반 평가(MBE: Model-Based Evaluation) 방법이

Corresponding Author: Rohae Myung. Department of Industrial Management Engineering, Korea University, Seoul 136-713.

Phone: 02-3290-3392, E-mail: rmyung@korea.ac.kr

Copyright@2011 by Ergonomics Society of Korea(pISSN:1229-1684 eISSN:2093-8462). All right reserved. 
설계되었다(Bennett et al., 1989). 공학적 모델과 원칙을 바탕으로 설계된 모델 기반 평가 방법은 제시된 수행 과제 를 얼마나 잘 해낼 수 있는가에 대한 정량적인 평가를 가능 하게 하였다(Kieras, 1996). 이러한 모델 기반의 평가 방 법은 인지 모델(Cognitive Model) 등의 사용자 모델을 통 하여 실제 사람에 의한 실험 없이도 사용자의 행동을 정량 적으로 예측가능하고, 이를 통해 인터페이스 간의 비교가 가능하여 평가에 활용될 수 있다는데 중요한 역할을 하고 있다(Ritter et al., 2001). 이러한 모델들은 인터페이스 간 의 비교를 통해 더 나은 인터페이스를 선택하거나 인터페 이스를 재설계하고 새롭게 개발하는데 있어서 피실험자를 섭외하고 훈련시킬 필요가 없으며, 인터페이스의 개발 주기 또한 단축할 수 있기 때문에 수백만 달러의 비용을 절감 시킬 수 있다(Gray et al., 1993). 또한 이러한 인지 모델 들은 인간과 컴퓨터의 상호작용(HCI: Human Conputer Interaction) 분야에서 지각(Perceptual), 인지(Cognitive), 운동(Motor)의 세가지 측면을 고려한 인지구조(Cognitive Architecture)를 바탕으로 구성되어 있기 때문에 이러한 인 지 모델을 기반으로 한 모델 기반의 평가 방법은 시스템을 평가하는데 유용한 정량적인 결과를 도출해주며, 이 결과는 인터페이스를 설계하는데 객관적인 자료로 활용되고 있다 (Olson \& Olson, 1990).

이러한 인간의 행동을 평가하는 대표적인 인지구조에는 GOMS family (Olson \& Olson, 1990, Card et al., 1996; John \& Kieras, 1996a, 1996b, Kieras, 1996, 1999), ACT-R(Anderson et al., 1998), Soar(Newell, 1990), EPIC(Kieras \& Meyer, 1997) 등이 있다.

이 가운데 특히 GOMS 모델은 모델링 과정이 쉽고 간단 하며 직관적이어서 국내외 $\mathrm{HCI}$ 분야에서 다양하게 사용되 어 왔다(Myung, 2004; Williams, 2005, Henrick, 2006, Back, 2010, Jeon, 2010).

\subsection{The GOMS model}

GOMS란 목표(Goal), 조작자(Operator), 방법(Method), 선택규칙(Selection Rule)의 약자이며, GOMS 모델은 인간 의 지각, 인지, 운동에 대한 이해를 기본으로 시스템과의 교 류를 모델링 함으로써, 시스템, 인터페이스, 작업에서의 수행 시간, 에러 그리고 수행 과정을 도출할 수 있다. GOMS 모델 은 인간에게 실제로 발생되는 내적, 외적 과정을 단순화 시 켜서 조작자들로 사용하고 있다. 조작자들은 실험을 통해 정 해진 시간을 파라미터로 가지고 있으며, 이 조작자들을 개인 의 경험이나 훈련에 의해 선택규칙에 맞춰 순차적으로 조합 되어 목표를 달성하게 된다.

GOMS 모델은 Card, Moran, Newell에 의해 최초로
CMN-GOMS (Card, Moran and Newell GOMS) 가 1980년 대 초에 개발되어, KLM-GOMS (Keystroke Level Model GOMS, Card et al., 1983), CPM-GOMS(Cognitive Perceptual Model GOMS, John, 1988), NGOMSL (Natural GOMS Language, Kieras, 1999) 에 이르는 발전을 거듭해 왔다. 각 GOMS 모델은 가지고 있는 고유의 장단점으로 인 해 쓰이고 있는 분야가 조금씩 차이가 있으나 GOMS 모델 자체가 가지는 적용 범위는 매우 크다고 할 수 있다. 특히 NGOMSL은 자연어(Natural Language)로 구조화된 컴퓨 터 프로그램 형태의 GOMS 모델로써 다른 모델에 비해 범 용 범위가 넓고 가장 많은 설계정보를 추출할 수 있는 장점 을 가진 GOMS 모델 방식이다.

NGOMSL은 Computational GOMS 모델로써 Kieras (1999)에 의해 개발된 GLEAN(GOMS Language Evaluation and Analysis)으로 기존의 LISP 컴퓨터 언어에 의 해 짜여진 모델을 좀 더 보편적으로 쓰이는 컴퓨터 언어인 $\mathrm{C}++$ 컴퓨터 언어로 프로그래밍되어 있으며, EPIC 모델의 구조(Architecture)를 기반으로 하였기 때문에 인간의 인지 와 수행을 시뮬레이션화 하는데 있어서도 정확도가 높아 질 수 있었다. GOMS 모델의 제한점이었던 인간의 에러 부분 이 추가된 EGLEAN(Error-Extended GLEAN)은 가장 최근에 연구된 기법으로 상호적인 제품 및 시스템에도 적용 가능한 모델 기법으로 평가되고 있다(Kieras, 1999). 이렇 게 발전된 GOMS 모델을 CGOMS (Computational-GOMS) 라고 한다.

일반적으로 GOMS 모델은 절차 모델(Procedure Model) 방법의 한 종류로써 다른 모델에 비해 상위 레벨 작업 평가 와 예측이 가능하고 사용이 용이하다는 장점을 가지고 있다. 또한 NGOMSL 모델은 다른 인지 모델에 비해 인간의 인지 과정에 대한 이해도를 깊이 요구하지 않고 자연어 기반으로 작성을 하기 때문에 일반 사용자들이 모델을 수립하기가 편 리하다. 그리고 GOMS 모델은 다른 모델들에 비해 작업 시 발생하는 순서와 시간을 직관적으로 보여주므로 작업분석 (Task Analysis)를 쉽게 할 수 있다. 즉, 직관성이 좋은 작 업분석 결과를 통해 분석하고자 하는 시스템 또는 인터페이 스에서 작업의 어떠한 부분이 시간이 오래 걸리고 인지부하 를 증가 시키는지 쉽게 알 수 있다. 따라서 다른 인지 모델 에 비해 GOMS 모델 사용자는 인간공학 또는 심리학 등의 지식수준이 낮더라도 결과해석이 쉽게 가능하다.

이러한 장점에도 불구하고 GOMS는 다음과 같은 제한점 이 존재한다(Olson \& Olson, 1990). (1) 비숙련자에게는 적 용되지 않으며, (2) 실수(error)를 고려하지 않고, (3) 인간 의 병렬 행위 과정이 반영되지 않아 실제 인지 과정과 차이 가 존재하며, (4) 정신적 작업부하, (5) 평가되는 시스템의 기능적 특성, (6) 사용자의 피로, (7) 인간의 개인별 성향 등 
을 고려하지 않는다.

뿐만 아니라, 1990년대의 인지공학자들로부터 제기된 GOMS 모델의 한계는 과거 80년대의 시스템으로부터 추 출된 마우스 등을 포함하고 있는 행위 조작자(operator)가 GOMS 개발 당시(outdated)의 파라미터 값들이 적용되고 있다는 점이다(Olson \& Olson, 1990).

또한 과거에 비해 사용자들의 컴퓨터 사용 역량이 증대되 고 있기 때문에 시스템에서 제공하는 정보를 효과적으로 활 용하며 반복적 사용을 통해 여러 기능을 복합적으로 조작 할 수 있는 능력 또한 향상되었다. 특히 시각적 정보를 탐 색하는 동시에 마우스와 같은 도구를 동시에 제어를 할 수 있는 능력의 향상 또한 빨라지고 있다. 이러한 동시제어를 눈-마우스 협응(병렬) 작용(Eye-Mouse Coordination) 이라고 하며, 이것은 눈-손 협응(병렬) 작용(Eye-Hand Coordination)과 같은 맥락에서 발생되고 있다(Smith et al., 2000, Beig et al., 2010). 하지만 앞서 언급한 기존의 GOMS 모델이 인간의 병렬 행위 과정을 설계할 수 없다는 제한점(Olson \& Olson, 1990)이 실제 사용자들(특히, 전문 가 수준의 사용자)를 반영하고 있는 모델이라는 GOMS 모 델의 특징과 모순된다.

그러므로 본 연구에서는 이러한 인간의 실제 행동에서 발생되는 눈-손 병렬 과정에 관한 기존 연구들을 토대로 GOMS 모델의 단점으로 지적되고 있는 순차적 구조를 수정 하여 눈-마우스 병렬 작용을 포함하는 행위 조작자를 제안 하고자 한다. 즉, 기존의 컴퓨터 기반 GOMS(CGOMS) 모 델의 단점인 병렬 행위를 포함한 행위 조작자를 제안하여 좀 더 정확하게 인지 행위를 예측할 수 있는 컴퓨터 기반 GOMS(CGOMS) 모델을 만들고자 한다.

\section{Method}

본 연구에서는 인간의 인지와 행위에 관련된 문헌 연구를 통한 GOMS 모델의 수정을 진행하였다. 특히 기존의 GOMS 모델이 제안하는 마우스 행위와 관련된 모델 구성 방법을 분 석하고 문제점을 도출하였다. 각 과정에 포함된 조작자들의 특성을 실제 사용자의 특성과 가깝게 모델을 재구성하였으 며, 이 결과가 실제 피실험자를 통해 추출된 수행 결과와 비교하여 두 모델의 적합성을 평가해 보고자 한다.

\subsection{Drag activities in existing GOMS model}

인간의 행위는 Table 1 의 GOMS 모델에 내재된 조작자 와 파라미터 값으로 구성된다. GOMS 모델의 조작자는 크
게 외적 조작자와 내적 조작자로 분류되며, 외적 조작자는 Manual, Vocal, Visual, Auditory 등으로, 내적 조작자는 Analyst Defined, Task Memory Access 등으로 인간의 활동을 구분한다. 이러한 조작자들은 여러 사용자들로부터 직접 관찰되거나 Olson \& Olson(1989)와 같은 기존 연구 자들에 의해 연구된 결과를 파라미터 값으로 적용하고 있다.

Table 1. GOMS operators

\begin{tabular}{c|c}
\hline GOMS Operator & msec \\
\hline Keystroke & 280 \\
\hline Type-in & N Keys*50 \\
\hline Click & 200 \\
\hline Double-click & 400 \\
\hline Release & 100 \\
\hline Point-to & 1,100 \\
\hline Home-to & 400 \\
\hline Look_for_object_whose & 1,200 \\
\hline Look_at & 200 \\
\hline Get_task_item_whose & 1,200 \\
\hline Recall_LTM_item_whose & 1,200 \\
\hline Store & 0.0 or 50 \\
\hline Deleate & 0.0 or 50 \\
\hline Verify & 1,200 \\
\hline Speak & N syllables* 150 \\
\hline
\end{tabular}

컴퓨터 환경 기반의 인터페이스 평가 시 빈번히 이용되 는 GOMS 모델의 조작자들을 살펴보면, 키보드를 치고 (Keystroke), 마우스를 움직이고(Point_to), 클릭(Single/ Double click, Hold_down, Release)하는 Manual 조작자 가 있으며, 물체를 보는(Look_for_object_whose, Look_at) Visual 조작자가 존재하며, 이 값들의 대부분은 KLMGOMS (Card et al., 1983; John \& Kieras, 1996a, 1996b) 모델의 조작자들에 의거한다(Kieras \& Knudsen, 2006).

기존의 GOMS 모델에 따르면 인간의 인지와 행위는 하나 씩 순차적으로 발생 된다는 가정을 전제로 하고 있다. 그러 나 인간의 활동을 인지와 행위로 분리시켜 생각하기 어려 우며, 특히 조작자 중에서 조차 안구의 움직임과 같은 외적 조작자 또한 정신작용의 고려가 요구됨으로 내적 조작자의 시간을 적용하도록 되어 있다(Kieras \& Knudsen, 2006). 이러한 기존의 GOMS 모델에 포함된 조작자와 방법을 사용하여 마우스에 관련된 행위 중 본 연구에서 제안한 드 래그 과정은 크게 4 가지 형태로 나눌 수 있다. 첫째, 드래 그 시작점과 끝나는 지점을 사용자가 알지 못하여 시각 탐 
색(Look_for_obect_whose)을 통해 찾아내야 하는 경우 이다(Drag activity (a) - - Look for start point and look for end point). 둘째, 드래그 시작점은 사용자가 알지 못 하나 끝나는 지점은 이미 알고 있어 드래그 시작점은 시각 탐색(Look_for_object_whose)을 통해 찾아내야 하지만 끝나는 지점은 단지 보기(Look_at)만 하면 되는 경우이 다(Drag activity (b) - Look for start point and look at end point). 이와 반대로 세 번째 경우는 드래그 시작점 은 알고 있으나 끝나는 지점은 모르고 있어 시작점은 단 지 보면(Look_at) 되지만 끝나는 지점의 경우 시각 탐색 (Look_for_object_whose)을 통해 찾아내야 한다(Drag activity (c) - Look at start point and look for end point). 넷째, 드래그 시작점과 끝나는 지점을 이미 알고 있어 두 지 점 모두 보기(Look_at)만 하면 되는 경우이다(Drag activity (d) - Look at start point and look at end point).

기존 GOMS 모델은 위의 4 가지 드래그를 수행하기 위 해서 공통적으로 6 단계 (step)를 순차적으로 거치게 된다. Figure 1(a) 과 같이 Drag activity (a)의 경우 시작 목표와 도착 목표를 사용자가 모르고 있다고 가정하여 움직여야 할 목표를 찾아 보는 것 (Step 1; Look_for_object_whose) 을 시작으로 목표의 위치로 마우스를 움직이고(Step 2; Point_to), 목표를 시작점으로 마우스 오른쪽 버튼을 누 른 채로 선택된 상태를 유지하고(Step 3; Hold_down), 그 뒤 새로운 도착 목표를 찾은 후 이를 보고(Step 4; Look_for_object_whose), 그 위치로 마우스를 이동시킨 후(Step 5; Point_to), 누르고 있던 마우스 오른쪽 버튼을 떼는 과정(Step 6; Release)으로 드래그 작업이 진행되며 총 $4,800 \mathrm{~ms}$ 이 소요된다.

Drag activity(b)와 (c)의 경우에 있어서는 시작 목표와 도착 목표를 중 한 가지 목표를 이미 알고 있다고 가정하여 목표를 찾는 과정이 생략된 채 보는 과정만이 필요로 하기 때문에 Drag activity(b)의 경우에는 step 4의 과정이 Drag activity (c)의 경우에는 step 2의 과정이 Look_at 과정으로 바뀌면서 전체 시간이 $4,800 \mathrm{msec}$ 에서 모두 $3,800 \mathrm{msec}$ 로
줄어든다. 그리고 Drag activity(d)의 경우에서는 시작 목표 와 도착 목표를 사용자가 이미 모두 알고 있다고 가정하여 두 번의 Look_for_object_whose 과정을 Look_at 과정으 로 바뀌면서 드래그 작업의 수행 시간이 $2,800 \mathrm{msec}$ 가 된다.

\subsection{Drag activities in revised GOMS model}

Fischer et al.(1986) 와 Gribble et al.(2002)의 연구에 따르면 마우스 조작에서는 시각적 탐색을 하는 동안 손이 동시에 움직이게 되는 시간적 공통 부분(Overlap)이 존재 하는 눈-손 병렬 과정이 일어난다. 그리고 이러한 시각적 탐색 과정은 손의 움직임에 비하여 조금 일찍 시작되며 (Smith et al., 2000, Bieg et al., 2010) 손의 움직임이 끝 나는 시점까지 진행된다고 한다(Mackenzie, 2002).

따라서 위의 연구 결과를 바탕으로 수정된 GOMS 모델 에 의한 드래그 작업에서는 Figure 1(b)에서 볼 수 있듯이 기존의 GOMS 모델에서 분석된 드래그 작업의 몇 개의 단 계가 수정된 GOMS 모델에서는 시각 탐색이 일어나는 동안 마우스의 조작(손의 움직임) 과정이 동시에 일어나며, 이러 한 손의 움직임이 끝나는 지점에서 시각 탐색 또한 완료되는 단계가 병렬적으로 발생하게 된다.

수정된 GOMS 모델의 드래그 과정을 기존의 GOMS 모델 과 비교해 보면, 기존의 GOMS 모델의 움직여야 할 목표를 찾는 단계(Step 1)와 찾은 목표로 마우스를 움직이는 단계 (Step 2)를 수정된 GOMS 모델에서는 눈-손 병렬 과정에 의하여 시각 탐색 조작자(Look_for_object_whose; 1,200 $\mathrm{msec}$ )가 실행된 후 얼마 되지 않아 손의 움직임에 관련된 조작자(Point_to; $1,100 \mathrm{msec}$ )도 동시에 실행되어 동시에 종료되는 단계(Revised step 1)로 수정하였다. 그 이후에 이루어지는 기존 GOMS 모델에서 목표를 시작점으로 마우 스를 누르는 단계(Step 3)는 수정된 GOMS 모델에서도 동일하게 적용되며(Revised step 2), 기존 GOMS 모델에 서 새로운 도착 목표를 찾는 단계 (Step 4)와 도착 목표로 마우스를 움직이는 단계(Step 5)는 수정된 GOMS 모델에

\begin{tabular}{|c|c|c|c|c|c|}
\hline \multicolumn{2}{|c|}{1200} & \multicolumn{2}{|c|}{2400} & 3600 & \multirow{2}{*}{700} \\
\hline Look_for_object_whose & Point_to & $\begin{array}{l}\text { Hold } \\
\text { down }\end{array}$ & Look_for_object_whose & Point_to & \\
\hline
\end{tabular}

(a) The progress timeline of existing GOMS model for drag activity(a)

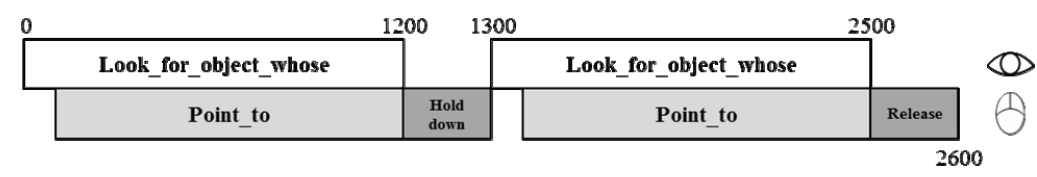

(b) The progress timeline of revised GOMS model for drag activity(a)

Figure 1. The progress timeline of drag activity(a) 
서 앞에서 적용된 시작점을 찾아 마우스를 가리키는 단계 (Revised step1)와 마찬가지로 눈-손 병렬 과정에 의하여 시각 탐색 조작자가 실행된 후 얼마 되지 않아 손의 움직임 에 관련된 조작자가 병렬적으로 실행되어 완료되는 하나의 단계(Revised step 3)로 수정하였다. 마지막 단계인 누르고 있던 마우스 오른쪽 버튼을 떼는 과정은 기존의 GOMS 모 델과 같다(Revised step 4).

기존의 GOMS 모델이 4 가지의 드래그 작업이 공통적으 로 6 개의 단계가 순차적으로 이루어지며 드래그 작업을 완 료하는데 걸리는 시간이 각각 $4,800 \mathrm{msec}$ (Drag activity (a)), 3,800msec (Drag activity (b), (c) ), 2,800msec (Drag $\operatorname{activity}(\mathrm{d}))$ 로 예측하는 것에 비해, 앞에서와 같은 과정에 의하여 드래그 작업을 위해 수정된 GOMS 모델은 눈-손 병렬 과정에 의해 시각 탐색 조작자와 손의 움직임에 관련된 조작자가 동시에 이루어져 전체적인 작업의 단계가 4 개의 단계로 줄어들며, 이로 인해 드래그 작업을 완료하는데 걸리 는 시간이 각각 2,600msec(Drag activity (a)), 2,500msec (Drag activity (b), (c)), 2,400msec(Drag activity(d))로 짧게 예측되었다.

\section{Verification Test}

\subsection{Participants}

본 실험에는 7 명 $(25.0 \pm 1.41$ 세 $)$ 의 피실험자가 자발적으 로 참여하였다. 이들은 모두 평균 6년 이상의 $\mathrm{PC}$ 및 마우스 사용 경험을 가지고 있으며, Microsoft Excel 2007 (평균 주 2회 이상 사용) 사용에 능숙한 전문가들로 이루어졌다.

\subsection{Task analysis and modeling}

본 실험에서는 컴퓨터 기반 시스템 중 통계분석을 하는데 이용되고 있는 Microsoft Excel 2007로 실험 과제를 구성 하였으며 이를 대상으로 검증을 실시하였다. 위와 같은 작업 은 GOMS 모델을 발전시키는데 중요한 기초 작업으로 인정 되어 최초의 GOMS 모델 개발에 적용된 바 있으며(John \& Kieras, 1996a), Microsoft Excel 2007의 경우 윈도우 기 반의 인터페이스답게 아이콘을 클릭하여 작업 실행이 가능 하도록 구성되어 있다.

실험 과제는 Microsoft Excel 2007에서 주어진 스프레 드 시트(Spread Sheet)에 입력된 X 값들과 Y 값들의 회귀 분석을 실행하는 것이다.

실험 과제로 선정된 회귀분석 과정은 크게 7 개의 하위 목표로 구성되어 있다. 첫 번째 하위 목표는 최상위 메뉴에
서 [Data; 데이터] 탭을 찾아 선택하는 것이고, 두 번째 하 위 목표는 데이터 메뉴 내의 [Data Analysis; 데이터 분석] 탭을 찾아 선택하는 것이다. 세 번째 하위 목표에서는 활성 화된 창에서 메뉴 바를 조정하여 [Regression; 회귀분석] 을 더블 클릭하여 선택하면 같은 위치에서 데이터 입력 창 으로 전환된다. 네 번째 하위 목표에서는 $\mathrm{y}$ 값을 드래그로 입력한다(y 값 입력 창이 활성화되어 있어 바로 입력이 가 능하다). 그러나 $\mathrm{x}$ 값 입력 시에는 $\mathrm{x}$ 값 입력 창이 활성화 되어 있지 않아 다섯 번째 하위 목표에서 $\mathrm{x}$ 값 입력 창을 선택한다. 여섯 번째 하위 목표에서는 $\mathrm{x}$ 값을 드래그로 입 력한다. 마지막 하위 목표에서는 [Ok; 확인] 버튼을 눌러 회귀분석 과제를 완료하게 된다.

회귀분석 과정에는 Figure 1에서 분석된 드래그 작업 이외에도 클릭 작업(Click activity)과 더블 클릭 작업 (Double-click activity)이 이용된다. 이러한 두 가지 마우 스 작업은 드래그 작업과 마찬가지로 기존 GOMS 모델에서 는 시각 탐색 과정과 마우스 조작이 순차적으로 구성된다. 하지만 클릭 작업과 더블 클릭 작업 또한 시각적 탐색을 하는 동안 손이 동시에 움직이게 되는 시간적 공통 부분이 존재하는 눈-손 병렬 과정이 일어나게 된다. 따라서 수정 된 GOMS 모델에서는 Figure 2에서와 같이 클릭 작업과 더블 클릭 작업을 각각 클릭 (Click <button〉) 과 더블 클릭 (Double_click <button〉)을 수행하기 전까지 공통적으로 목 표를 찾는 과정(Look_at) 과 마우스를 움직여 목표를 가리키 는 과정 (Point_to)을 병렬적으로 구성하였다. 기존 GOMS 모델에서 클릭 작업과 더블 클릭 작업을 각각 $1,500 \mathrm{msec}$, $1,700 \mathrm{msec}$ 로 예측하는 것에 비해, 수정된 GOMS 모델에 서는 각각 $1,300 \mathrm{msec}, 1,500 \mathrm{msec}$ 로 짧게 예측되었다.

본 연구에 적용되는 조작자들은 기본적으로 내재되어 있 는 파라미터 값(default value)를 유지한다는 가정하에 각 작업 단계에 맞춰 기존의 GOMS와 수정된 GOMS 모델에 따라 구성하도록 하였다.

\begin{tabular}{|l|l|l|}
\hline \multicolumn{2}{|c}{200} & 1300 \\
\hline Look_at & Point_to & Click \\
\hline
\end{tabular}

(a) Click activity for existing GOMS

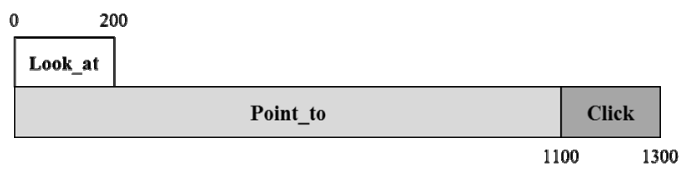

(b) Click activity for revised GOMS

Figure 2. The progress timeline of click activity 


\subsection{Results}

본 연구에서는 기존의 NGOMSL 모델에 눈-손 병렬 과 정의 적용을 통해 수정된 GOMS 모델을 제안하였다. 실제 수행 시간과 GOMS 모델에 의한 예측 결과의 통계적 차이 를 검증하기 위해 Student's t-test를 수행하였다. 그 결과 실제 실험 참가자들의 수행 시간과 기존의 GOMS 모델의 결과는 유의한 차이를 나타내었으며 $(p-$ value $=0.0086)$, 수정된 GOMS 모델 결과와도 유의한 차이를 보였다 $\left(p^{-}\right.$ value $=0.0078)$. 이는 Figure 3 에서도 볼 수 있듯이 실험 과제를 수행하는데 있어서 각 개인 간의 편차가 매우 크며, 평균 수행 시간을 기준으로 하였을 때 수정된 GOMS 모델 이 기존의 GOMS 모델에 비하여 인간의 수행 시간을 좀 더 유사하게 예측하고 있으나 아직까지 인간의 수행 시간을 유의한 차이가 없이 예측하지 못한다고 할 수 있다. 다시 말하면 수정된 GOMS 모델의 예측값이 실제 측정값 보다 과대 평가되었으며 실제 실험 참가지들의 Microsoft Excel 2007의 회귀분석 과제에 대한 수행도는 균일하지 않고 2 단계(2 level)로 이루어져 있다고 볼 수 있다.

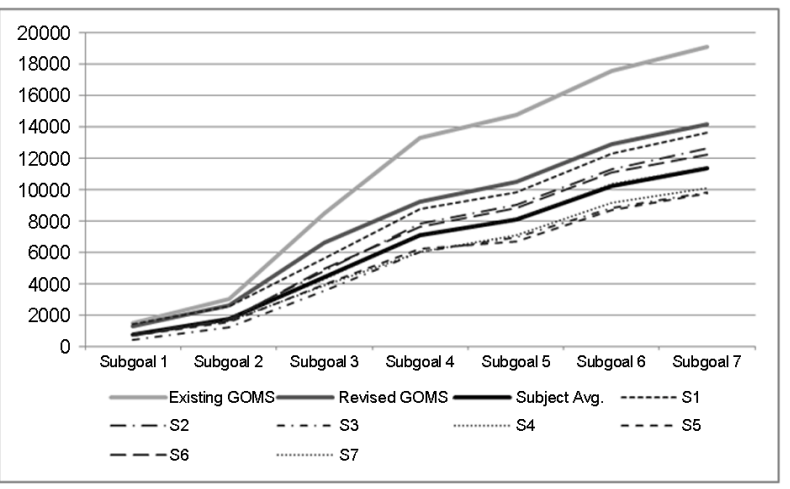

Figure 3. Cumulative performance time
Table 2에서 보면 기존의 GOMS 모델에서는 총 수행 시 간을 $19,100 \mathrm{msec}$ 로 예측하였으며, 이는 실제 피실험자의 총 수행 시간 $11,622 \mathrm{msec}$ 와는 약 $70.0 \%$ 의 오차율이 발생 하였다. 그러나 수정된 GOMS 모델에서는 총 수행 시간이 $14200 \mathrm{msec}$ 로 예측되어 실제 피실험자와의 오차율은 약 $22.2 \%$ 로 감소되었다. 수정된 GOMS 모델이 인간의 인지와 행동 특성인 눈-손 병렬 과정을 반영하여 기존의 GOMS에 비해 오차율이 크게 감소하여 실제 인간의 수행 시간에 근접 하였으나 약 2.59초 과대 평가되었다. 수정된 GOMS 모델 의 예측값이 실제 실험 참가자의 수행값과 통계적으로 차 이를 보이나, Olson \& Olson(1990)에 의하면 유효 모델로 서의 오차율의 범위 (rule of thumb)를 $20 \%$ 내외로 본다고 하므로 $22.2 \%$ 의 오차율을 보이는 수정된 GOMS 모델은 유효하다고 할 수 있다.

실험 과제를 구체적으로 살펴보면 각 하위 목표들은 드 래그 작업과 클릭 작업으로 반복되고 있다. 기존의 GOMS 모델의 경우 각각의 클릭 작업을 $1,500 \mathrm{msec}$ 로, 수정된 $\mathrm{GOMS}$ 모델의 경우 $1,300 \mathrm{msec}$ 로 동일하게 예측하고 있다. 그러나 실제 피실험자의 수행 시간은 첫 번째 클릭 작업은 $1,017 \mathrm{msec}$, 두 번째 클릭 작업은 $966 \mathrm{msec}$, 세 번째 클릭 작업은 $980 \mathrm{msec}$, 마지막 클릭 작업은 $1,116 \mathrm{msec}$ 로 모두 다르다. 이 4 번의 클릭 작업의 평균 시간은 $1,020 \mathrm{msec}$ 으로 기존의 GOMS 모델이나 수정된 GOMS 모델이 상당히 과대 평가됐음을 알 수 있다. 이러한 실제 피실험자의 수행 시간 의 차이를 보이는 이유는 각각의 하위 목표에서 수행되는 클릭 작업에서의 마우스 이동 거리가 각각 다르기 때문이다.

또한, 드래그 작업의 경우 네 번째 하위 목표와 여섯 번째 하위 목표에서는 수정된 GOMS 모델이 각각 $2,500 \mathrm{msec}$, $2,400 \mathrm{msec}$ 로 실제 피실험자의 수행 시간인 $2,662 \mathrm{msec}$, $2,178 \mathrm{msec}$ 와 유사하게 예측하고 있다. 그러나 세 번째 하 위 목표에서의 드래그 작업은 실제 피실험자의 수행 시간 $(1,715 \mathrm{msec})$ 에 비하여 수정된 GOMS 모델이 약 0.79초

Table 2. Regression analysis task using Microsoft Excel 2007(msec)

\begin{tabular}{|c|c|c|c|c|}
\hline \multicolumn{2}{|c|}{ Main goal; Method_for_goal: Regression analysis } & Existing GOMS & Revised GOMS & Empirical Data \\
\hline \multicolumn{2}{|l|}{ Subgoal 1; Select data menu(Click activity) } & 1,500 & 1,300 & 1,017 \\
\hline \multicolumn{2}{|c|}{ Subgoal 2; Select data analysis menu(Click activity) } & 1,500 & 1,300 & 966 \\
\hline \multirow{2}{*}{ Subgoal 3; Select regression analysis menu } & Drag activity(b) & 3,800 & 2,500 & 1,715 \\
\hline & Double-click & 1,700 & 1,500 & 988 \\
\hline \multicolumn{2}{|l|}{ Subgoal 4; Input Y-data(Drag activity(a)) } & 4,800 & 2,600 & 2,662 \\
\hline \multicolumn{2}{|c|}{ Subgoal 5; Select input box for X-data(Click activity) } & 1,500 & 1,300 & 980 \\
\hline \multicolumn{2}{|l|}{ Subgoal 6; Input X-data(Drag activity(d)) } & 2,800 & 2,400 & 2,178 \\
\hline \multicolumn{2}{|l|}{ Subgoal 7; Select Ok button(Click activity) } & 1,500 & 1,300 & 1,116 \\
\hline \multicolumn{2}{|l|}{ Total } & 19,100 & 14,200 & 11,622 \\
\hline
\end{tabular}


과대 예측하고 있다. 이는 클릭 작업에서와 마찬가지로 드래 그 작업에서 수행되는 손의 이동 거리가 다르며, 세 번째 하 위 목표에서의 드래그 작업이 컴퓨터 환경에서 이용되는 드 래그 작업의 평균 이동 거리에 비해 짧은 것이 원인으로 분 석된다.

이러한 클릭 작업과 드래그 작업에 대한 수행 시간 예측이 비정확한 것은 정해진 파라미터를 사용하여 이동 거리에 따 른 수행 시간을 반영하지 못하는 기존 GOMS 모델의 제한 점에 기인한다고 할 수 있다.

\section{Discussion}

본 연구에서는 기존의 NGOMSL 모델에 눈-손 병렬 과 정의 적용을 통해 수정된 GOMS 모델을 제안하였다. 수정 된 GOMS 의해 예측된 총 수행 시간은 실제 사람의 수행 시간과 비슷하게 단축되기는 하였으나 아직까지 완벽하게 인간수행 시간을 도출해 내지는 못하고 있다.

이러한 차이의 원인은 다음과 같이 생각할 수 있다. 첫째, GOMS 모델의 전제 조건인 수행도의 전문가 레벨을 정량적 으로 정의하기가 어렵기 때문이다. 본 연구에서도 물리적인 실험 참가자들의 Microsoft Excel 2007에 대한 전문성(나 이, 컴퓨터 사용 연한, Microsoft Excel 2007 사용 빈도수) 을 균질(Homogeneous) 로 간주하고 실험을 하였으나 결과 적으로 참가자들의 수행도의 차이가 두 집단으로 나누어짐 으로써 수정된 GOMS 모델과 실제 수행 시간과 차이를 발 생시켰기 때문이다.

둘째, 눈-손 병렬 과정을 반영하고 있기는 하지만 기존의 조작자에 내재된 파라미터 값을 기본으로 하고 있기 때문에 실제로 발생되는 인지 과정과 행위 과정을 완벽하게 구현해 내지 못한다는 점이다. 상황에 따른 인지 과정을 반영하지 못한 채 모두 동일한 시간을 적용해야 함은 물론 시각 탐색 에서도 동일한 시간을 적용하여 판단하는 것을 기본으로 하 고 있기 때문에 인간에게 발생되는 복잡한 정보 처리 과정을 정확히 묘사하는 것이 부족하다. 예를 들면 본 연구에서 제 안한 드래그 작업 중 수행 시간이 가장 짧은 드래그 작업 (d)의 경우에는 두 번의 인지 과정(Look_at) 과 두 번의 행 동(Point_to)으로 구성되어 있는데 이를 수정된 GOMS 모 델에서 눈-손 병렬 작용 통하여 드래그 작업을 수정하였으 나 조작자의 내재된 파라미터 값이 이미 정해져 있으며 행 동(Point_to) 조작자의 시간이 길어 아무리 빠르게 하여도 $2,400 \mathrm{msec}$ 보다 작을 수 없게 된다. 그러나 본 연구에서 측 정된 드래그 작업의 실험적 결과값의 평균은 $2,200 \mathrm{msec}$ 인 데, 이는 수정된 GOMS 모델에서 제안된 드래그 작업의 최
소값인 2,400msec보다 작다. 즉, 이는 행동(Point_to) 조 작자의 내재된 파라미터 값이 과대 평가되어 있다고 할 수 있으며, 따라서 행동(Point_to) 조작자의 값을 거리에 따라 이동 시간이 달라지는 Fitts' law 값이 반영된 값을 적용하지 않는 한 더 이상의 정확한 예측은 불가능 하다고 생각된다.

셋째, 이렇게 조작자에 내재된 모든 파라미터는 1980년 대 Card et al.(1983), Olson \& Nilsen(1988), John \& Newell(1989) 등에 의해 관찰되고 결정된 값들을 기본으 로 하고 있다. 하지만 수십 년이 지난 지금, 컴퓨터는 삶의 일부가 되었고 그 만큼 많은 사람들이 컴퓨터 작업에 익숙해 졌다. 그러므로 실제 인지 과정과 행동의 수행 시간은 기존 의 GOMS 모델에 내재되어 있는 파라미터 값들보다 빨라졌 을 것이다. 또한 컴퓨터 환경 또한 사용자에게 친숙해 지고 성능적으로도 빠른 수행능력을 보이고 있기 때문에 현재의 시스템 환경에서 새롭게 추출된 파라미터의 적용이 보다 정 확한 수행 시간을 예측할 수 있을 것이라 생각된다.

이와 같은 조작자와 파라미터의 수정은 Henrik(2006), Amant et al. (2007), Lee \& Myung (2008) 등에 의해 연구 되었으며, Lee \& Myung(2008)이 제안한 모델에 의거하여 수행 시간을 예측하면 회귀분석 과제는 $12,660 \mathrm{~ms}$ (오차율 약 $11.35 \%$ ) 가 도출되어 본 연구에서 제안된 수정된 GOMS 모델에 비해서도 더 정확하다. 하지만 조작자 정의에 기반이 된 가설이 모바일 기기에 한정되어 있기 때문에 컴퓨터 기 반의 환경에서도 적용이 될 수 있는지는 추후 연구가 필요 하다.

\section{Conclusion}

본 연구에서는 기존의 GOMS 모델의 규칙에 따라 모델을 구성하고 문헌 연구를 통해 GOMS 모델에서의 제한적이었 던 인간의 인지 과정과 행위를 반영하기 위한 조작자의 구 성을 병렬적으로 배치하여 모델을 수정하였다. 또한 그에 상 응하는 설계 정보를 통해 실제 작업과 비교함으로써 새로운 결과들을 분석해내는 것으로 진행되었다.

이에 기존의 GOMS 모델에 비해 예측 수행 시간의 정확 도가 높아진 수정된 GOMS 모델을 구현할 수 있었으며, 검 증실험 결과를 통해 수정된 GOMS 모델이 실제 인간의 수 행 시간을 잘 예측할 수 있음을 확인하였다.

다양한 컨트롤러를 갖는 인터페이스의 평가를 위한 Computational-GOMSL 수정 모델 제안은 사용성 평가 뿐 만 아니라 새로운 시스템을 제안하는 개발자 입장에서도 매 우 중요한 역할을 한다.

따라서 본 연구는 대표적으로 사용된 마우스와 클릭/드래 
그형 인터페이스뿐만 아니라, 보다 역동적이고 상호적인 제 품 및 시스템에서도 적용 가능한 GOMS 모델링 기법으로의 전환의 계기를 마련하고 GOMS 모델의 확장과 향상을 위한 가이드라인으로 제시될 수 있다.

\section{Acknowledgements}

본 논문은 2008 년 한국한술진흥재단의 기초연구지원 기 초과학 단독연구(Grant No. KRF-2008-313-D01233) 와 2단계 BK21사업 지원을 받아 수행된 것임.

\section{References}

Anderson, J. R. and Lebiere, C., Atomic Components of Thought, Lawrence Erlbaum Associates, New Jersey, London, 1998.

Back, J., Myung, R. and Yoon, D., Applying CPM-GOMS Analysis for predicting and Explaining Two-Handed Korean Text Entry Task on Mobile Phone, Human Factors and Ergonomics Society Annual Meeting Proceedings, Human Performance Modeling, 977-981, 2010.

Bennett, J., Butler, K. and Whiteside, J., Usability Engineering, A tutorial presented at CHI, '89 Conference on Human Factors in Computing Systems, Austin, TX, April 30-May 5, 1989.

Bieg, H., Reiterer, H. and Bulthoff, H. H., Eye and Pointer Coordination in Search and Selection Tasks, ETRA2010, Austin, TX, March 22-24, 2010.

Bothell, D., ACT-R 6.0 Reference Manual. From the ACT-R Web site: http://act-r.psy.cmu.edu/actr6/reference-manual.pdf, 2007.

Card, S. K., Moran, T. P. and Newell, A., Computer Text-Editing: An Information-Processing Analysis of a Routine Cognitive Skill, Cognitive Psychology, 12, 32-74, 1980b.

Card, S. K., Moran, T. P. and Newell, A., The Psychology of HumanComputer Interaction, L. Erlbaum Associates, 1983.

Fischer, B. and Rogal, L., Eye Hand Coordination in Man: A Reaction Time Study, Biological Cybernetics, 55, 253-261, 1986.

Galitz, W., The Essential Guide to User Interface Design, 2nd ed., Wiley, 2002.

Gillan, D. J., Holden, K. and Adam, S., How does Fitts' Law Fit Pointing and Dragging?, Proceedings of the SIGCHI conference on Human factors in computing systems, New York, 227-234, 1990.

Gould, J. D., Boies, S. J., Levy, S., Richards, J. T. and Schoonard, J., The 1984 Olympic Message Systme: A Test of Behavioral Principles of System Design. Communications of the ACM, 758-769, 1987.

Gould, J. D. and Lewis, C., Designing for Usability: Key Principles and What Designers Think., Communications of the ACM, 300-311, 1985. Gray, W. D., Spohrer, J. C. and Green, T. R. G., End-user programming languages: The CHI '92 workshop report., SIGCHI Bulletin, 25(2), 46-50, 1993.

Gribble, P. L., Everling, S., Ford, K. and Mattar, A., Hand Eye Coordination for Rapid Pointing Movements, Experimental Brain Research, 145, 372-382, 2002.

Henrik, T., Measuring Website Usability for Visually Impaired People - A Modified GOMS Analysis, Proceedings of the 8th International ACM SIGACCESS Conference on Computers and Accessibility, Portland, Oregon, 55-62, 2006.

Holleis, P., Otto, F., Hubmann, H. and Schmidt, A., Keystroke-Level Model for advanced Mobile Phone Interaction, Proceedings of the SIGCHI Conference on Human Factors in Computing Systems, San Jose, California, 1505-1514, 2007.

Ivory, M. Y. and Hearst, M. A., Improving Web Site Design, IEEE Internet Computing, vol. 6, no. 2, 56-63, 2002.

Jeon, Y. J., Revised GOMS Operator for Drag and Drop, Proceedings of the Human Factors and Ergonomics Society 54th Annual Meeting, San Francisco, California, 1742-1746, 2010.

John, B. E., Contributions to Engineering Models of Human-Computer Interaction, Doctoral Dissertation, Canegie Mellon University, Pittsburgh, 1988.

John, B. E., "Why GOMS?", ACM Interaction, NY, USA, 2(4), 80-89, 1995.

John, B. E. and Newell, A., Cumulating the Science of HCI: From S-R Compatibility to Transcription Typing, ACM SIHCHI Bulletin, 20, 109-114, 1989

John, B. E. and Kieras, D. E., Using GOMS for User Interface Design and Evaluation: Which Technique?, ACM Transactions on ComputerHuman Interaction, 3(4), 287-319, Dec, 1996a.

John, B. E, and Kieras, D. E., The GOMS Family of User Interface Analysis Techniques: Comparison and Contrast, ACM Transactions on Computer-Human Interaction, 3(4), 320-350, $1996 \mathrm{~b}$.

Kieras, D. E., A Guide to GOMS Model Usability Evaluation using NGOMSL, The Handbook of Human-Computer Interaction. 2nd edition, North-Holland, Amsterdam, 1996.

Kieras, D. E., A Guide to GOMS Model Usability Evaluation using GOMSL and GLEAN3, Technical report, University of Michigan, Ann Arbor, MI., 1999.

Kieras, D. E. and Knudsen, K., Comprehensive Computational GOMS Modeling with GLEAN. In Proceedings of BRIMS 2006, Baltimore, May 16-18, 2006.

Kieras, D. E. and Meyer D. E., An Overview of the EPIC Architecture for Cognition and Performance with Application to Human-Computer Interaction, Human-Computer Interaction, 12, 391-438, 1997.

Kieras, D. E., Scott, D. W. and Meyer D. E., Predictive Engineering Models Using the EPIC Architecture for a High-Performance Task, Proceedings of the SIGCHI Conference on Human Factors in Computing Systems, 11-18, Denver, Colorado, United States, 1995.

Landauer, T. K., The Trouble With Computers: Usefulness, Usability, and Productivity. MIT Press, 1995.

MacKenzie, I. S., Movement time Prediction in Human-computer Interfaces, Human-Computer Interaction (2nd ed.), 483-493. Kaufmann, Losaltos, 
CA., R. M. Baecker, W. A. S. Buxton, J. Grudin and S. Greenberg (Eds.), 1992.

MacKenzie, I. S. and Soukoreff, R. W., Text Entry for Mobile Computing: Models and Methods, Theory and Practice, Human-Computer Interaction, 17, 147-198, 2002.

Myung, R., Keystroke-level Analysis of Korean Test Entry Methods on Mobile Phones, International Journal of Human-Computer Studies, 60, 545-563, 2004.

Newell, A., Unified Theories of Cognition, Harvard University Press, Cambridge, 1990.

Olson, J. R. and Nilsen, E., Anlaysis of the Cognition Involved in Spreadsheet software Interaction, Human-Computer Interaction, 3, 309-350, 1988.

Olson, J. R. and Olson, G. M., The Growth of Cognitive Modeling in Human-Computer Interaction since GOMS, Human-Computer Interaction, 5, 221-265, 1990.

Parush, A., Nadir, R. and Shtub, A., Evaluating the layout of graphical user interface screens: Validation of a numerical, computerized model. International Journal of Human Com-puter Interaction, 10(4), 343 $-360,1998$

Puerta, A. and Maulsby, D., MOBI-D: A Model-Based Development Environment for User-Centered Design, Proceedings of $\mathrm{CHI}$ '97, Atlanta, 4-5, 1997.

Ritter, F. E, Shadbolt, N. R., Elliman, D., Young, R., Gobet, F. and Baxter, G. D., Techniques for Modeling Human Performance in Synthetic Environments: A Supplementary Review, Wright-Patterson Air Force Base, 2001.

Salvucci, D. D. and Goldberg, J. H., Identifying Fixations and Saccades in Eye-Tracking Protocols, Proceedings of the EyeTracking Research and Applications Symposium, Palm Beach Gardens, Florida, 71-78, 2000.

Smith, B. A., Ho, J., Ark, W. and Zhai, S., Hand Eye Coordination Patterns in Target Selection, Proceedings of Eye Tracking Research \& Applications Symposium 2000, Palm Beach Garden, FL, USA, 2000.

Takahiro, H. and Kazuyuki, S, Different Memory Types for Generating Saccades at Different Stages of Learning, Neuroscience Research, 55(3), 271-284, 2006.

Viviani, P., Baud-Bovy, G. and Redolfi, M., Perceiving and Tracking Kinaesthetic Stimuli: Further Evidence of Motor Perceptual Interactions, Journal of experimental psychology, 23(4), 1232-1252, 1997.
Whisenand, T. G. and Emurian, H. H., Analysis of cursor movements with a mouse, Proceedings of HCI International '97, San Francisco, USA 533-536, 1997.

Wiecha, C. and Boles, S., Generating User Interfaces: Principles and Use of It Style Rules, Proceedings of the Third Annual ACM SIGGRAPH Symposium on User Interface Software and Technology, 21-30, 1990.

Williams, K. E., Computer-aided GOMS: A Description and Evaluation of a Tool that Integrates Existing Research for Modeling HumanComputer Interaction, International Journal of Human-Computer Interaction, 18, 39-58, 2005.

\section{Author listings}

Yongho Lee: cash30yh@korea.ac.kr

Highest degree: BE in Department of Industrial System Information Engineering, Korea University

Areas of interest: HCI, Cognitive Modeling

Young Joo Jeon: young_jeon@korea.ac.kr

Highest degree: BA, School of Art and Design, Korea University Areas of interest: $\mathrm{HCI}$

Rohae Myung: rmyung@korea.ac.kr

Highest degree: Ph.D., Industrial Engineering, Texas Tech University Position title: Professor in Dept. of Industrial Engineering, Korea University

Areas of interest: HCI, Cognitive Modeling

Date Received : 2011-02-09

Date Revised : 2011-03-14

Date Accepted : 2011-03-16 